\title{
Modeling microevolution in a changing environment: The evolving quasispecies and the Diluted Champion Process
}

\author{
Ginestra Bianconi†, Davide Fichera $\ddagger$ Silvio Franz $\ddagger$, and Luca \\ Peliti@|| \\ † Physics Department, Northeastern University, Boston MA 02115 USA \\ $\ddagger$ Laboratoire de Physique Théorique et Modèles Statistiques, CNRS \\ and Université Paris-Sud, UMR 8626, Bât. 100, 91405 Orsay cedex France \\ $\S$ Dipartimento di Scienze Fisiche and Sezione INFN, Università "Federico II", \\ Complesso Monte S. Angelo, 80126 Napoli Italy
}

\begin{abstract}
Several pathogens use evolvability as a survival strategy against acquired immunity of the host. Despite their high variability in time, some of them exhibit quite low variability within the population at any given time, a somehow paradoxical behavior often called the evolving quasispecies. In this paper we introduce a simplified model of an evolving viral population in which the effects of the acquired immunity of the host are represented by the decrease of the fitness of the corresponding viral strains, depending on the frequency of the strain in the viral population. The model exhibits evolving quasispecies behavior in a certain range of its parameters, and suggests how punctuated evolution can be induced by a simple feedback mechanism.
\end{abstract}

PACS numbers: $87.23 . \mathrm{kg}, 87.10 . \mathrm{Mn}$

E-mail: peliti@na.infn.it

|| To whom correspondence should be addressed. 


\section{Introduction}

Pathogenic viruses, in order to survive and successfully reproduce have to fight against the immune system of their host organisms. Some viruses use evolvability as a successful strategy to escape acquired immunity. In the presence of adaptive response in the host newly arising mutants can acquire a competitive advantage with respect to the wild type. Neverthless, in some viral populations one often observes "quasispecies" behavior, in which individuals are strongly similar to one another.

A prototypical example of this behavior is exhibited by the Influenza A virus, which makes use of high antigenical variability (genetic drift) to escape acquired immune response. Virus strains circulating in different epidemic seasons, in spite of eliciting a certain amount of cross-response by the immune system of the hosts, mutate fast enough to be able to infect the same host several times in the course of its life. However in any given observed epidemics, the viral population is sufficiently concentrated around a well defined strain that effective vaccines can be prepared. Moreover, antigenic changes are punctuated: antigenic assays show that the sequences of the dominant circulating type H3N2 fall into temporally correlated clusters with similar antigenic properties ([1]). This behavior, which has been described as an evolving viral quasispecies, contrasts with the prediction of naive models of viral evolution (see, e.g., [2]), where the interaction with the immune system leads to proliferation of strains with different antigenic properties and, consequently, to the impossibility of preparing effective vaccines. It has recently been experimentally shown [3] that some bacteria in a changing environment can develop a genotype which produces highly variable phenotypes, presumably adapted to different environments. One may speculate whether such a bet-hedging strategy is available to viruses, due to the small size of their genetic material and their high mutation rate, especially for RNA-based viruses.

A different example is the one of the in-host evolution of the HIV, which exploits high mutation rates to counter the immune adaptation of its host (see, e.g., [4, 5]). Despite its high mutation and substitution rates, the viral population shows low levels of differentiation during most of the asymptomatic phase.

Several solutions have been proposed to this evolutionary puzzle, based on the analysis of mathematical models. In [6], elaborating on previous theory ([7]), it was proposed that in the case of influenza A, a short-time strain-trascending immunity could give account for the quasispecies behavior. It was observed in [8] that this shortterm immunity would be ineffective in the absence of heterogeneities in infections, due either to the structure of network of infective contacts or to variations in the infective efficacy of different strains. An alternative explanation was put forward in [9] based on the analysis of a model, where the heterogeneity of the neutral phenotypic network of the evolving virus is taken into account. An important feature in influenza epidemics seems to be the directionality of its propagation. According to the analysis of [10] and [11] yearly epidemics typically start from seeds in South-East Asia before spreading

worldwide. This suggests the presence of strong bottlenecks in the viral population that 
could lead to relatively small effective population sizes and to a strong reduction of genetic variability.

Models can suggest mechanisms that can produce the observed evolutionary pattern. However, even the simplest individual based model of viral evolution in a host population ([8]) is too complicated to lead to a detailed exploration of parameter space. It is not clear which conditions on evolutionary and epidemiological parameters allow for the evolving quasispecies behavior. In particular we will be interested to the dependence on the population size. We wish to ascertain whether in the presence of the infectivity reduction due to the immune response of the host, the quasispecies behavior can appear for some parameter ranges in the infinite population, or is only possible in sufficiently small populations. This last possibility would emphasize the importance of population bottlenecks in the ecology of the virus.

In this paper we introduce a simplified model of evolving viral population that keeps into account the interaction between virus and host immunity in an effective way. The effectiveness of reproduction of a given viral type at a given time depends on its age and its past frequency in the host population. We get a model that is simple enough to allow to study at least numerically, the dependence on the different parameters, and in particular on the population size. Our findings suggest that quasispecies behavior and punctuated equilibria are only possible for small enough populations, whereas, if the model parameters are not scaled with the population size, one always has proliferation in the large population limit.

The organization of the paper is the following: in section 2 we define our model as an extension of Kingman's house of cards model, where strains are represented by self-reproducing entities and the immune memory of the host is taken into account by the decrease of the strain fitness with time. In this model, the fitness of a mutant is independent of that of its parental strain. This assumption is justified in our approach by the fact that since the parental fitness decreases very quickly due to the immune adaptation of the host, we do not expect long-lived fitness correlations to exist among related strains. In section 3 we recall some known results about the dynamics of the model in the absence of memory. In section 4 we study the effect of the immune memory in numerical simulations and show the existence of the evolving quasispecies regime. In 5 we analyze in more detail the behavior of the system in the evolving quasispecies regime. In 6 we introduce a simple effective stochastic process that helps in understanding the dynamical behavior of the quasispecies regime. Finally some conclusions are drawn.

\section{The Model}

We consider here a model of an evolving viral population of constant size consisting of $N$ individuals. The population evolves according to a Wright-Fisher process for asexually reproducing populations $([12,13])$. At each discrete time step $t$ the population reproduces and is completely replaced by its progeny. Each individual is characterized by its strain label $S$. The expected offspring size of an individual belonging to strain $S$ 
is proportional to the Wrightian fitness $w_{S}(t)$ of its strain. The fitness $w_{S}(t)$ of a strain $S$ depends on its intrinsic fitness $w_{S}^{0}$, proportional to its basic reproductive number, and changes with time as described below. In what follows we shall also use the notation $w_{S}=\mathrm{e}^{f_{S}}$ and call $f_{S}$ the Fisher fitness of $S$. As it is well known ([14]) the Wright-Fisher model can be described as a process where each individual $j$ at generation $t+1$ "chooses" its parent $i$ at generation $t$ with probability equal to $w_{S_{i}}(t) / \sum_{j=1}^{N} w_{S_{j}}(t)$. Consequently, for large $N$, the offspring size of an individual $i$ is a random Poissonian variable with average $w_{S_{i}}(t) /\langle w\rangle_{t}$.

At each reproduction event, a mutation can take place with probability $\mu$. Upon mutation, a new strain $S^{\prime}$ appears, and its intrinsic fitness $w_{S^{\prime}}^{0}$ is drawn from a fixed probability distribution $\rho(w)([8,15,16,17])$, independently for each mutation event: this corresponds to the infinite allele approximation where no back mutations are possible. The fitness $w_{S^{\prime}}$ of the newly formed strain is equal to its intrinsic fitness $w_{S^{\prime}}^{0}$. If fitnesses did not depend on time, the model would coincide with Kingman's house-of-cards model ([15]).

The effect of acquired immunity of the host population on the viral reproduction is represented by letting the fitness $w_{S}$ of each strain $S$ decrease at each generation with a rate proportional to the number $N_{S}(t)=N n_{S}(t)$ of individuals belonging to that strain in the population. For simplicity we consider an exponential decrease of the fitness:

$$
w_{S}(t+1)=w_{S}(t) \mathrm{e}^{-h N_{S}(t) / N}
$$

where $h$ is a parameter which determines the rate of fitness decrease.

In the following we will describe mainly the case in which $\rho(w)$ is a log-normal distribution $\rho(w) \propto \mathrm{e}^{-\log ^{2} w / 2 a^{2}} / w$. We have also investigated the model with the uniform fitness distribution finding the same qualitative results.

\section{Dynamic behavior in the absence of immunity}

We start by reviewing the behavior of the model for $h=0$, i.e., in the absence of immune memory of the host $([15,17,18])$. In this case, in the limit of large populations $N \rightarrow \infty$, it is possible to derive an exact equation for the evolution of the fraction $x_{t}(w)$ of individuals with fitness $w$ at time $t$, namely

$$
x_{t+1}(w)=\frac{w}{\langle w\rangle_{t}}(1-\mu) x_{t}(w)+\mu \rho(w),
$$

where $\langle w\rangle_{t}=\int \mathrm{d} w w x_{t}(w)$. The nature of the solution of this equation depends on the properties of $\rho(w)$. If $\rho(w)$ has a compact support (i.e., $\rho(w)=0$ for $w>w_{\max }$ ), the equation admits a stationary state satisfying

$$
x(w)=\frac{\mu \rho(w)}{1-(1-\mu) w /\langle w\rangle_{t}} .
$$

This distribution is analogous to the Bose distribution, with $w$ playing the role of the Boltzmann factor. Therefore, if $\int \mathrm{d} w \rho(w) /\left(1-w / w_{\max }\right)<\infty$, the Einstein condensation can take place. This transition is known as the error threshold in 
evolutionary models, and separates a poorly adapted phase at high mutation rate from a well adapted phase at low mutation rate. In the well adapted (condensed) phase, found for $\mu<\mu_{\mathrm{c}}$, a finite fraction $\nu$ of the population has the maximum fitness $w_{\max }$. Here $\nu$ and $\mu_{\mathrm{c}}$ are given by

$$
\nu=1-\frac{\mu}{\mu_{\mathrm{c}}} ; \quad \mu_{\mathrm{c}}^{-1}=\int_{0}^{w_{\max }} \mathrm{d} w \frac{\rho(w)}{1-w / w_{\max }} .
$$

In this phase the reproduction rate of the individuals with maximal fitness equals one: $(1-\mu) w_{\max } /\langle w\rangle=1$, while all the others have reproduction rates smaller than one. Conversely, in the poorly adapted (uncondensed) phase, less than maximally fit individuals can reproduce. If the integral $\int \mathrm{d} w \rho(w) /\left(1-w / w_{\max }\right)$ is divergent no error threshold appears.

The situation where the fitness distribution $\rho(w)$ extends to infinity is more complex and interesting. In this case, equation (2) does not admit a stationary solution and one obtains instead a run-away. However, in contrast with the case of compact support, the dynamics for a finite population with $N \gg 1$ individuals can now be substantially different from the infinite population limit. In fact, the evolution process can be described as a non stationary record process where condensates of a given fitness are replaced by ones of higher fitness ([18]), with persistence times that becomes longer and longer as the fitness increases. It has been noticed in [17] that, however, an uncondensed phase can be metastable for long times. Let us denote by $w_{\text {mut }}(t)$ the maximum fitness appearing among the mutants in $t$ generations. As at each time step a number $N \mu$ of new mutants appear, the maximum fitness $w_{\text {mut }}(1)$ for one generation satisfies

$$
N \mu \int_{w_{\text {mut }}(1)}^{\infty} \mathrm{d} w \rho(w) \simeq 1 .
$$

If the reproduction rate $(1-\mu) w_{\text {mut }}(1) /\langle w\rangle$ is smaller than one, the uncondensed phase will be stable. This quantity depends on $t$ via the average $\langle w\rangle$. Then the stability time can be determined by the condition that the maximal fitness of mutants over an

interval of $t$ generations, as determined by the relation $N \mu t \int_{w_{\text {mut }}(t)}^{\infty} \mathrm{d} w \rho(w) \simeq 1$ has a reproduction rate equal to one. In this way one gets an effective $N$ - and $t$ - dependent error threshold, which can be approximately described by equations

$$
\nu=1-\frac{\mu}{\mu_{\mathrm{c}}} ; \quad \mu_{\mathrm{c}}^{-1}=\int_{0}^{w_{\mathrm{mut}}(1)} \mathrm{d} w \frac{\rho(w)}{1-w / w_{\text {mut }}(t)} .
$$

In figure 1 we compare this analysis with numerical simulations for the lognormal distribution with parameter $a=0.3$, showing the fraction $\nu$ of the condensate for two independent populations of $N=1000$ individuals evolving for $t=5000$ generations, together with the predictions of equation (6) as a function of the mutation rate.

\section{Introducing the fitness decay}

Let us now study the effect of the fitness decay and set $h>0$. We continue to dwell mainly on the case of lognormal fitness distribution $\rho(w)$ with parameter $a=0.3$. In 


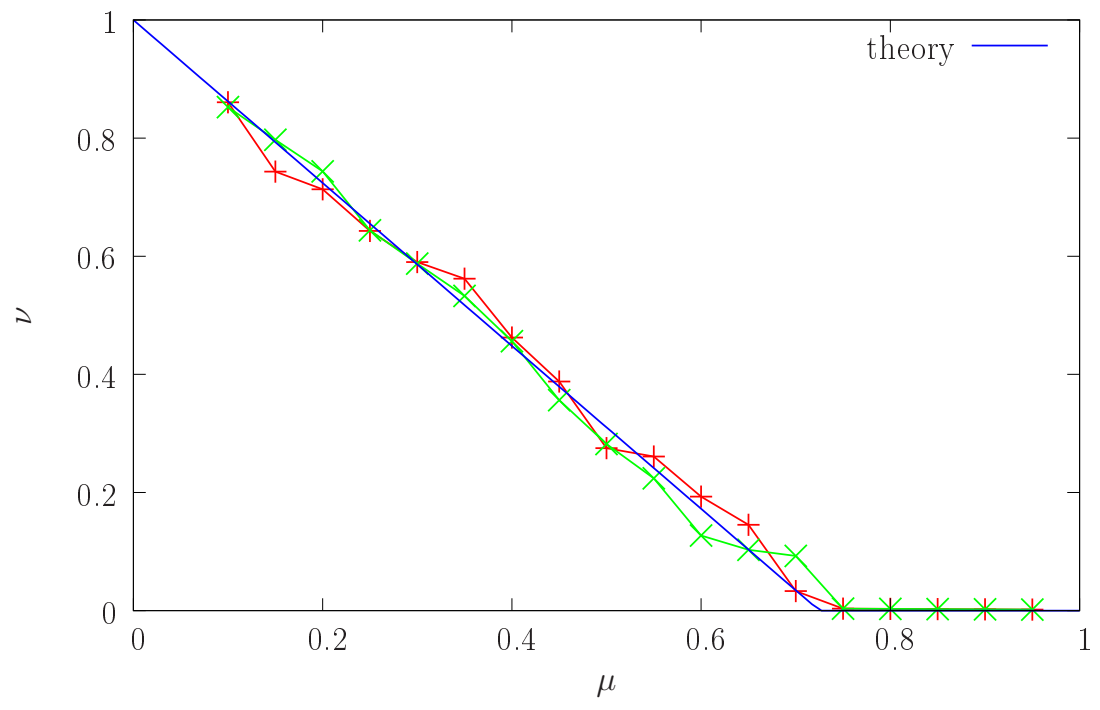

Figure 1. Condensate fraction $\nu$ as a function of the mutation rate $\mu$ for two independent populations of 1000 individuals evolving for $t=5000$ generations, together with the theoretical result. The data display a well defined error threshold in agreement with the theory.

order to study the possibility of a non zero condensate we observe that while for $h=0$ the maximally occupied genome should usually coincide with the one of highest fitness, this is not necessarily true if $h>0$. Let us define therefore, at any given time, the leader strain in the population as the one that is populated by the largest fraction of individuals, and the $\max$ as the one whose fitness is largest. In figure 2 we plot the occupation fraction $\nu$ of the leader as a function of the mutation rate, for several values of $h$, in a population of size $N=1000$. The figure shows that while the leader occupation fraction is a decreasing function of $h$, the error threshold is not destroyed by the immunity response and the critical value $\mu_{\mathrm{c}}$ is roughly independent of $h$. We also observe that a non zero $h$ introduces a natural time scale into the system, and one should expect that stationarity is reached after a finite relaxation time, so that the critical value of $\mu$ becomes time independent. In figure 3 we compare the fitness of the leader as a function of time in the condensed phase for $h=0$ to the one corresponding to a small value of $h$. One can manifestly see that while stationarity does not hold in the former case, it holds in the latter. In figure 4 we plot the occupation of the leader as a function of the mutation rate for various values of $N$. One can see that the error threshold slowly moves to higher values of $\mu$ as $N$ increases. This also appears in the behavior of the leader fitness $w_{\text {lead }}$ and of the maximal fitness $w_{\max }$ in the system which exhibit a maximum at the error threshold. We plot these quantities together with the average fitness in figure 5. In order to obtain a less cluttered plot, the fitnesses are rescaled by the factor $f(N)=\exp \left(\sqrt{2 a^{2} \log \left(N / \sqrt{2 \pi a^{2}}\right)}\right)$, where $a=0.3$ is the width of the distribution of $\log w_{0}$, expected on the basis of extreme-value statistics for the lognormal distribution. The range of variation of this factor is too small to warrant 


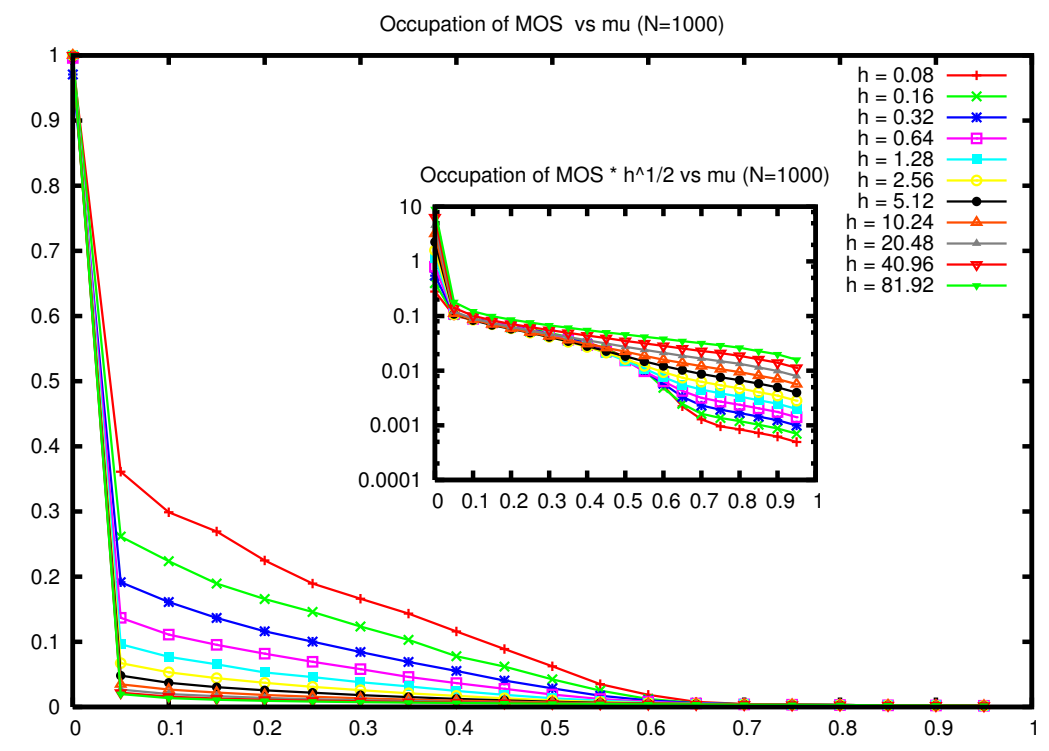

Figure 2. Occupation fraction $\nu$ of the leader for $N=1000, a=0.3$ and several values of $h$, as a function of $\mu$. The error threshold takes place at an $h$-independent value of $\mu$ if $h$ is not too large. In the inset we plot $\nu h^{1 / 2}$ exhibiting the proportionality of $\nu$ to $h^{-1 / 2}$ in the condensed phase.

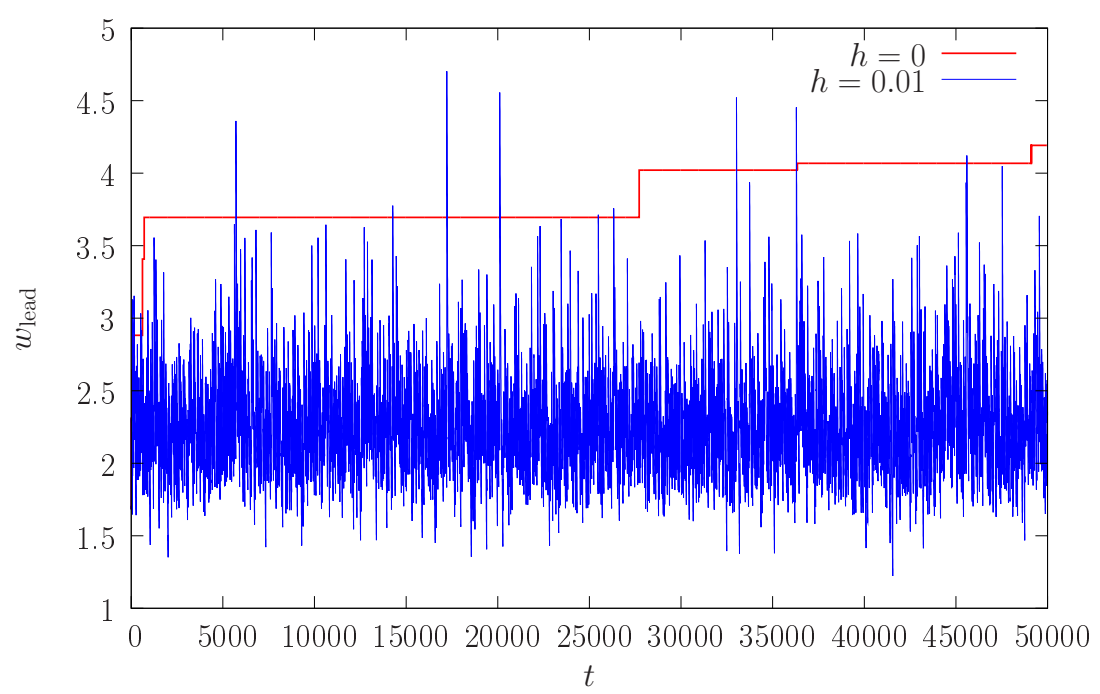

Figure 3. Fitness $w_{\text {lead }}$ of the leader as a function of time for the lognormal fitness distribution with $a=0.3, \mu=0.1$, and for $h=0$ and $h=0.01$ respectively. For $h=0$ the fitness follows a record process increasing on average with time. As soon as $h>0$ the process becomes stationary.

drawing any conclusion from this observation. Notice that while the leader and the maximal fitnesses reach the maximum at the error threshold, the average fitness has a maximum at a lower value of $\mu$. Close to the maximum of the average fitness the leader and the max occupation fractions turn out to be roughly independent of $N$, as it can 


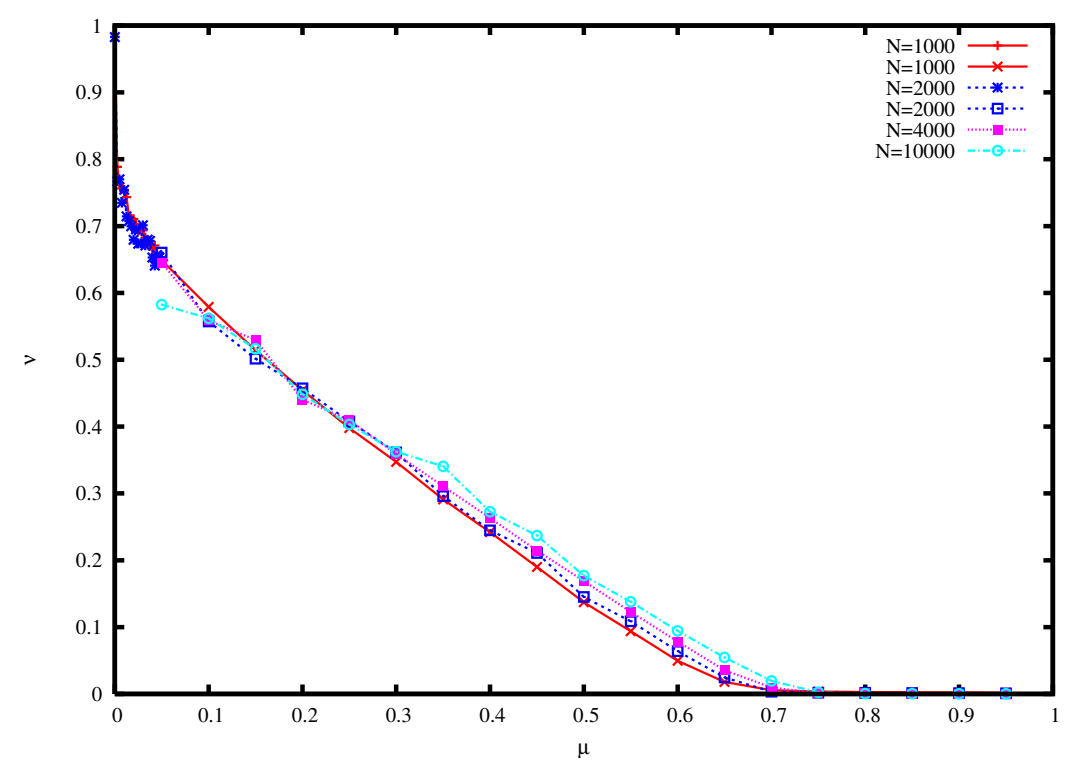

Figure 4. Occupation $\nu$ of the leader for $h=0.01$ as a function of $\mu$ for $N=$ $1000,2000,4000,10000$. The error threshold slowly moves to higher values of $\mu$ as $N$ increases.

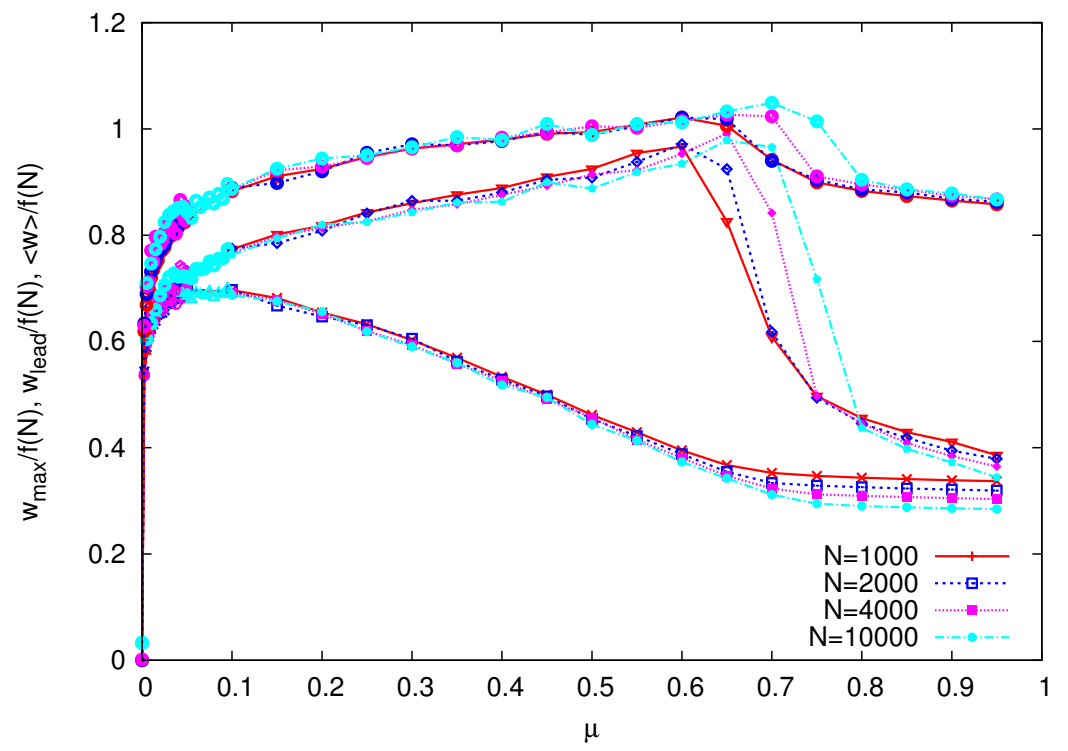

Figure 5. Maximal $w_{\max }$ (upper line), leader $w_{\text {lead }}$ (middle line) and average $\langle w\rangle$ (lower line) fitness for $h=0.01$, as a function of $\mu$, for $N=1000,2000,4000,10000$. The fitnesses are rescaled by the factor $f(N)=\exp \left(\sqrt{2 a^{2} \log \left(N / \sqrt{2 \pi a^{2}}\right)}\right)$, where $a=0.3$ is the width of the distribution of $\log w_{0}$, expected on the basis of extremevalue statistics. The error threshold is slowly displaced to higher values of $\mu$ as $N$ increases.

be seen from figure 6, which plots these two quantities as a function of $h$. Conversely, figure 7 shows that the fitness of the leader and of the $\max$ are functions of $h / N$ : for 
larger $N$ a proportionally larger $h$ is needed to decrease the fitness of the same amount.

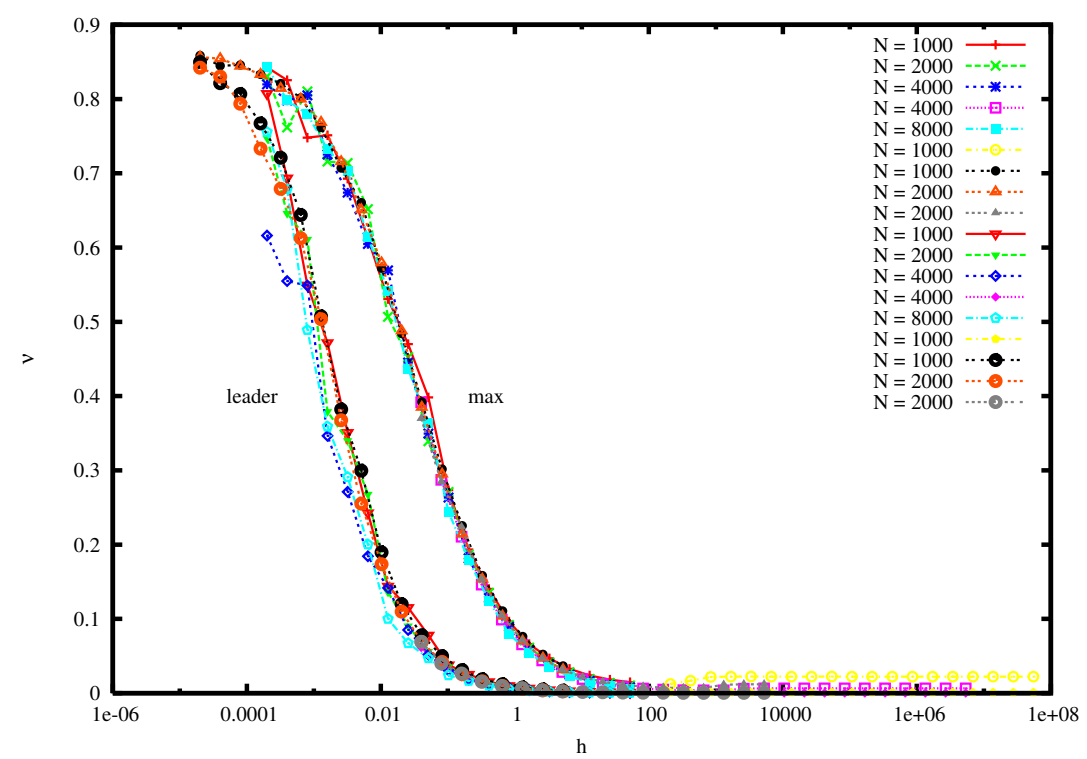

Figure 6. Occupation of leader (upper) and max (lower) as a function of $h$ for $\mu=0.1$ and various values of $N=1000,2000,4000,8000$.

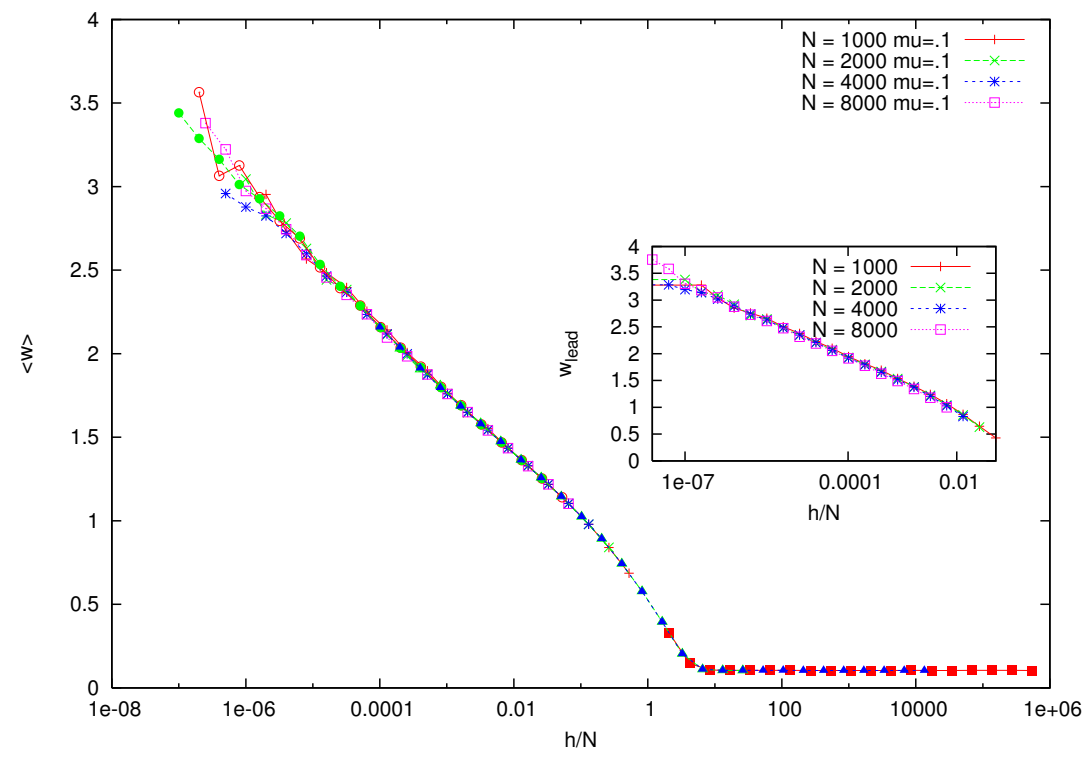

Figure 7. Average (main panel) and leader (inset) fitness as a function of $h / N$ for $\mu=0.1$ and various values of $N=1000,2000,4000,8000$. 


\section{Dynamics}

The results of the previous section clearly show that the error threshold persists even in the presence of a small amount of immune response in the host. In order to gain a better understanding of the evolving quasispecies regime characterizing the well adapted phase, let us look at the temporal behavior of the system. In figure 8 we show the occupation fractions of the max and of the leader as a function of time, in the well adapted phase with $\mu=0.1$ and $h=0.01$ in a population of $N=1000$ individuals. We can see that both quantities exhibit a regular behavior in time, which can be qualitatively described as follows. When a new fitter mutant establishes in the population, it substitutes the previous leader and remains leader for a while before being at his turn substituted. We find therefore a kind of punctuated equilibrium dynamics, as announced in the introduction. Figure 9 shows the corresponding behavior of the average fitness of the
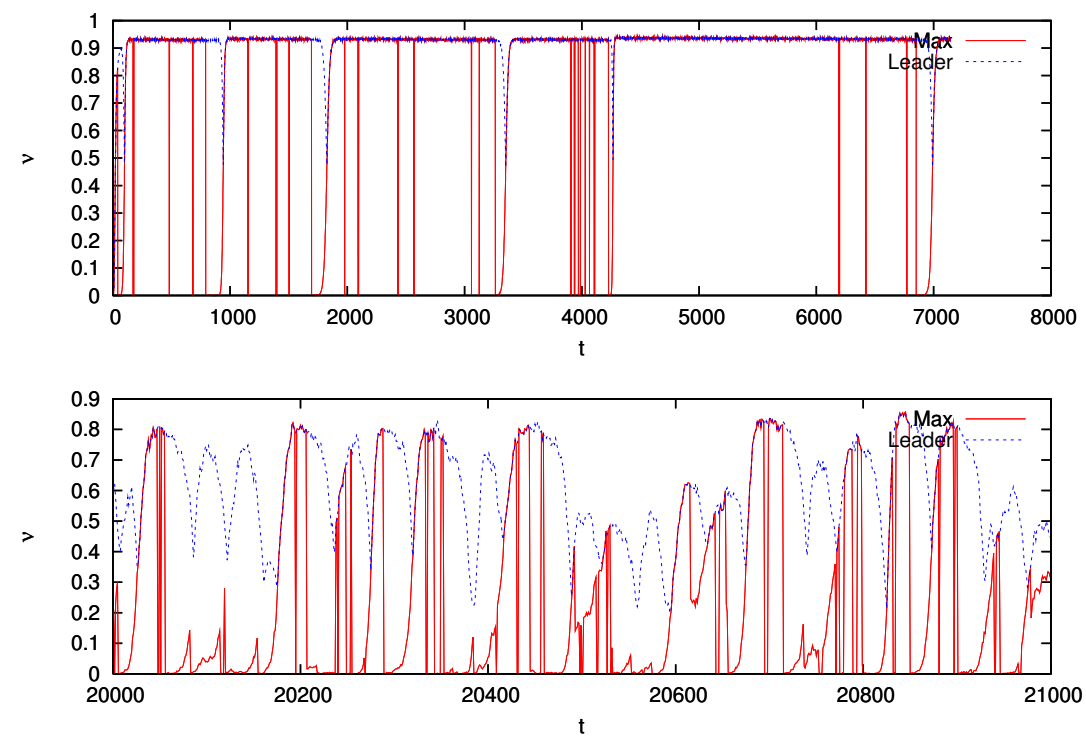

Figure 8. Time dependence of the occupation fraction of the max and of the leader in the evolving quasispecies regime. Upper panel: the system is far from the threshold, fluctuations are small, the substitution time is the only random quantity, $N=40.000$, $\mu=0.05, h=0.01$. Lower panel: the system is close to the error threshold, fluctuations are large, $N=1000, \mu=0.1, h=0.01$.

leader and of the max respectively. One sees that the fitness of the max stays constant for a while after the emergence of the mutant, and then, when the max replaces the leader, it rapidly decreases until a new leader takes over. It is interesting to look at the reproduction rate of the leader strain. As shown in figure 10, this is observed to differ substantially from one only close to substitution events. Just before substitution, the challenged leader has a reproduction rate which is smaller than one, while it is larger then one just after substitution. In the periods when the leader largely dominates the population (dominance periods) the reproduction ratio is very close to one, and diversity 

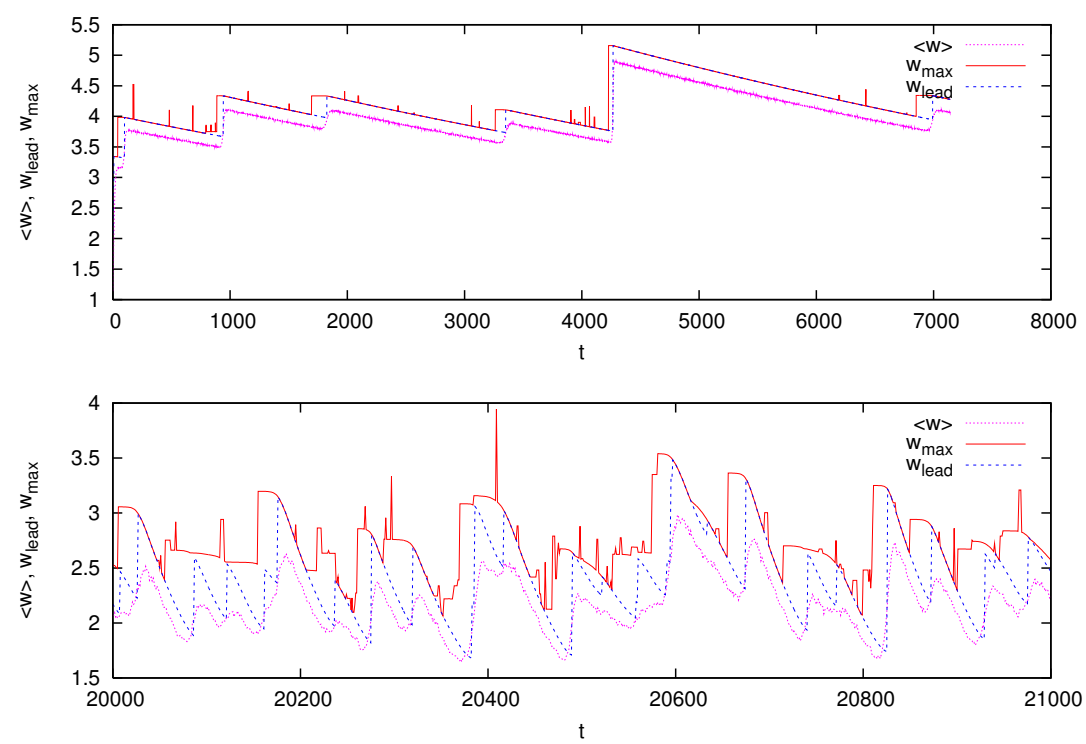

Figure 9. Behavior of the fitness in the quasispecies regime. Green: max; red: leader. For both panels the parameters are the same as in the corresponding ones of figure 8

in the population is maintained by mutants. We measured the average duration $t_{\text {subs }}$ of
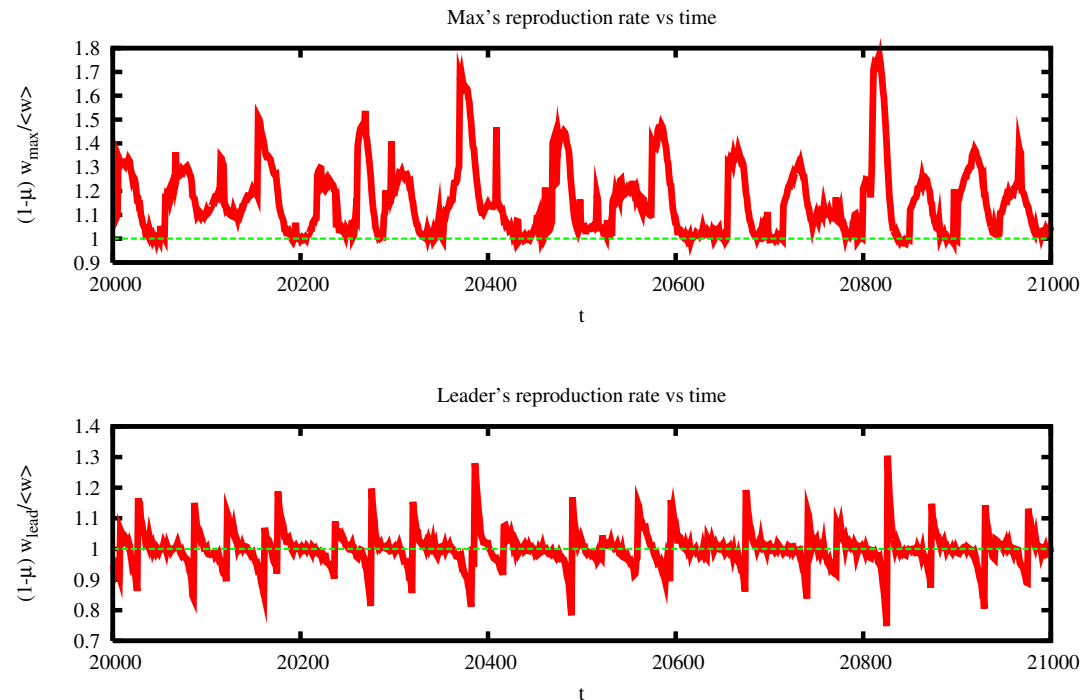

Figure 10. Reproduction rate of the max and of the leader, for parameters corresponding to the lower panels of 8 and 9 . Notice that the reproduction rate of the max is always larger than 1 , while the reproduction rate of the leader is noticeably different from one only close to a substitution event.

the leadership, i.e., the time interval between successive substitutions. In figure 11 we plot $\left(t_{\text {subs }}-1\right) h^{1 / 2}$ vs. $h$, showing that this quantity it is roughly proportional to $h^{-1 / 2}$, and essentially independent of $\mu$, in the quasispecies regime. Given the natural decay 
time of fitness on time scales of order $1 / h$ it would have been natural to hypothesize a substitution time of the same order. Our numerical result show that substitutions being separated by times of order $h^{-1 / 2}$ are much more frequent.

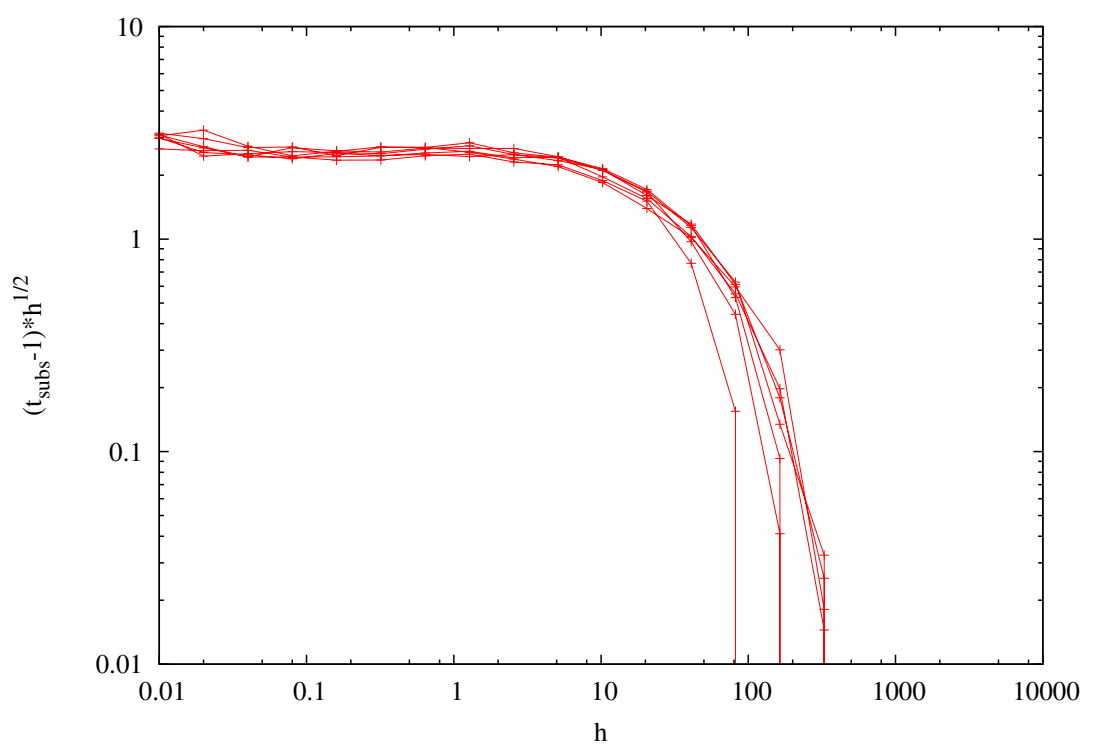

Figure 11. Plot of $\left(t_{\text {subs }}-1\right) h^{1 / 2}$ as a function of $h$ for $\mu=$ $0.05,0.1,0.15,0.2,0.25,0.3,0.35$. The curves are roughly constant in the quasispecies regime.

\section{The Diluted Champion Process}

Certain aspects of the evolving quasispecies dynamics at low $h$ and $\mu$ can be interpreted by introducing an effective stochastic process for the substitution. We start by defining the Champion Process where at each time there is a leader. The performance of the leader, which is represented by a fitness value, declines exponentially in time: $W_{t+1}=\lambda W_{t}$ with $\lambda<1$. The leader is challenged at regular intervals of time, and the challenger's fitness is extracted randomly from a distribution $\rho^{*}(W)$. If the fitness of the challenger exceeds the one of the leader in charge, the challenger substitutes the old leader. Otherwise there is no substitution and the old champion remains in charge. The Diluted Champion Process (DCP) is a simple variation of the process where the substitution process is not deterministic. Substitutions can occur with a certain probability that depends on the fitness ratio between the challenger and the champion in charge. This model can obviously be adapted to competition situations where the performances of the individuals naturally decline in time.

Now, in the evolving quasispecies regime of our model, while the substitution times and the leaders fitnesses are random quantities, the dynamics between punctuations is essentially deterministic. Moreover, the champion substitution process requires times that are much shorter than the typical times of change of the fitness. Indeed as figures 8 
and 9 show, the fitness of the champion starts to decay as soon as the new challenger takes over. These observations make the DCP description pertinent for our model. During the dominance periods, at each time step the champion is challenged by a number of mutants $M$ Poisson distributed with $\langle M\rangle=N \mu$, with random fitnesses drawn from $\rho(w)$. By elementary extreme values statistics, the best fit challenger has therefore a fitness distribution given by $\rho^{*}(w)=\mu N \rho(w) \mathrm{e}^{-\mu N \int_{w}^{\infty} d w^{\prime} \rho\left(w^{\prime}\right)}$. This will have a fixation probability $f\left(w / w_{\text {leader }}\right)$, which can be estimated by the Kimura expression: $f(x)=\left(1-x^{-1}\right) /\left(1-x^{-N}\right)$. Thus the probability of survival in one round of a leader with fitness $w_{\text {leader }}$ is given by

$$
\Phi\left(w_{\text {leader }}\right)=1-\int \mathrm{d} w \rho^{*}(w) f\left(\frac{w}{w_{\text {leader }}}\right) .
$$

At this point we will assume, consistently with the simulations, that for small $h$ we can neglect the fixation time with respect to the leader strain lifetime, and suppose that for a champion $w_{t+1}=\lambda w_{t}$, with a constant $\lambda$ equal to $\lambda=\exp \left(-n^{*} h\right)$ where $n^{*}$ is the condensate fraction. In the case of the DCP, one can safely assume $n^{*} \simeq 1$, leading to $\lambda=\mathrm{e}^{-h}$.

It is possible to formally write the basic formula for the probability that a champion with initial fitness $w_{0}$ is substituted after exactly $t+1$ time steps by a new champion with fitness $w_{1}$, namely:

$$
P_{t}\left(w_{1} \mid w_{0}\right)=\prod_{s=1}^{t} \Phi\left(w_{0} \lambda^{s}\right) \rho^{*}\left(w_{1}\right) f\left(\frac{w_{1}}{w_{0} \lambda^{t}}\right) .
$$

From this equation all the the properties of the DCP can be in principle derived. One can express the conditional probability that the leader fitness equals $w_{1}$ given the previous leader fitness $w_{0}$ by

$$
M\left(w_{1} \mid w_{0}\right)=\sum_{t=0}^{\infty} P_{t}\left(w_{1} \mid w_{0}\right) .
$$

Then one can in principle obtain the stationary distribution of the leader fitness $\mu(w)$ from

$$
\mu\left(w_{1}\right)=\int \mathrm{d} w_{0} M\left(w_{1} \mid w_{0}\right) \mu\left(w_{0}\right)
$$

and the distribution of the substitution time at stationarity from

$$
q(t)=\int \mathrm{d} w_{0} d w_{1} P_{t}\left(w_{1} \mid w_{0}\right) \mu\left(w_{0}\right) .
$$

Unfortunately these equations are not easily analyzed, so in order to test the validity

of this simple effective model we had to simulate it. In figure 12 we show the typical evolution of the leader's fitness as a function of time. The DCP clearly reproduces the qualitative features of our model.

\section{Conclusions and perspectives}

We have introduced a simple effective model describing the evolution of a population of pathogens in the presence of the immunological response of the host. The model is 

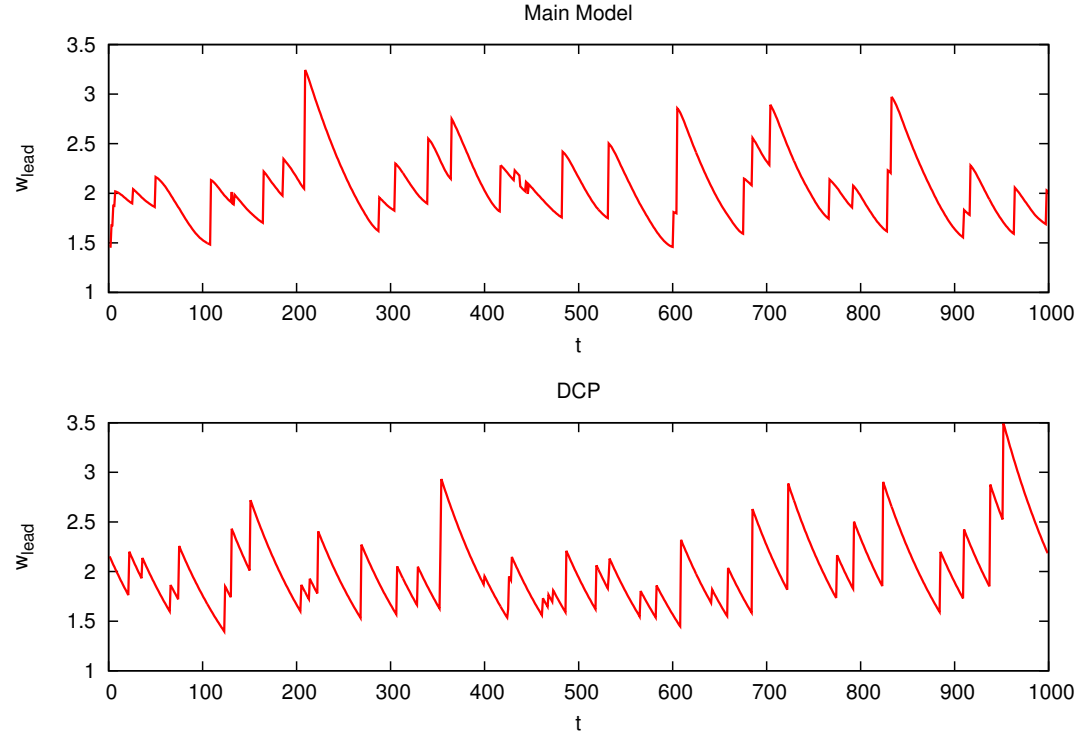

Figure 12. Leader's fitness as a function of time for the main model and for the DCP with $N=400, \mu=0.05, h=0.01$ and $a=0.3$. The DCP clearly reproduces the qualitative features of the main model.

simple enough to be amenable to a systematic numerical investigation, and allows one to identify a parameter region in which the coexistence of a well-defined quasispecies and of a fast turnover of the dominant strain is be quite robust. The behavior of the model in this region can be understood in terms of a further simplified model: the Diluted Champion Process. Our model can be interpreted in several ways: on the one hand, as describing the evolving viral strains present in the host population, e.g., in the case of the annual influenza epidemics; on the other hand, as the evolution of the viral population in a chronic infection of a single host individual, as in intra-host HIV evolution. In this case, fluctuations in the strength of immune response could cause proliferation of the number of strains and then the failure of the immunity system to downregulate all the viral strains (see, e.g., [19]). It is possible to obtain a more realistic description of this regime by, e.g., relaxing the fixed population constraint. It is also possible to consider a higher degree of correlation in the fitness landscape, in order to understand the extent of the stability of the punctuated equilibrium regime.

We are working on a generalization of our model taking into account the existence of different regions. They can be understood as different climatic regions in the case of epidemics, or localization in different organs in the case of intra-host infection. This investigation will allow us to understand better the different roles played by different regions: seeder, reservoirs, etc., as observed in recent analyses of epidemiological data collected in the last years $([10,11])$, or to identify possible gene-surfing phenomena in the diffusion of new strains from external regions ([20]). 
Microevolution in a changing environment

\section{Acknowledgments}

LP acknowledges the support of FARO.

\section{References}

[1] Smith D J, Lapedes A S, de Jong J C, Bestebroer T M, Rimmelzwaan G F, Osterhaus A D M E and Fouchier R A M 2004 Science 305 371-376

[2] Girvan M, Callaway D S, Newman M E J and Strogatz S H 2002 Phys. Rev. E 65031915

[3] Beaumont H J E, Gallie J, Kost C, Ferguson G C and Rainey P B 2009 Nature 462 90-93

[4] Liu S L, Rodrigo A G, Shankarappa R, Learn G H, Hsu L, Davidov O, Zhao L P and Mullins J I 1996 Science 273 415-416

[5] Shankarappa R, Margolick J B, Gange S J, Rodrigo A G, Upchurch D, Farzadegan H, Gupta P, Rinaldo C R, Learn G H, He X, Huang X L and Mullins J I 1999 J. Virol. 73 10489-10502

[6] Ferguson N M, Galvani A P and Bush R M 2003 Nature 422 428-433

[7] Webster R G, Bean W J, Gorman O T, Chambers T M and Kawaoka Y 1992 Microbiol. Mol. Biol. Rev. 56 152-179

[8] Tria F, Lässig M, Peliti L and Franz S 2005 JSTAT: J. Stat. Mech.: Theory and Experiment $\mathrm{P} 07008$

[9] Koelle K, Cobey S, Grenfell B and Pascual M 2006 Nature 314 1898-1903

[10] Rambaut A, Pybus O G, Nelson M I, Viboud C, Taubenberger J K and Holmes E C 2008 Nature 453 615-619

[11] Russell C A, Jones T C, Barr I G, Cox N J, Garten R J, Gregory V, Gust I D, Hampson A W, Hay A J, Hurt A C, de Jong J C, Kelso A, Klimov A I, Kageyama T, Komadina N, Lapedes A S, Lin Y P, Mosterin A, Obuchi M, Odagiri T, Osterhaus A D M E, Rimmelzwaan G F, Shaw M W, Skepner E, Stohr K, Tashiro M, Fouchier R A M and Smith D J 2008 Science 320 340-346

[12] Wright S 1931 Genetics 16 97-159 reprinted in Bull. Math. Biol. 52, 241-295 (2006)

[13] Fisher R A 1930 The Genetical Theory of Natural Selection (Oxford University Press) reprinted 1958: Dover Publications, New York

[14] Derrida B and Peliti L 1991 Bull. Math. Biol. 53 355-382

[15] Kingman J F C 1978 J Appl Prob 15 1-12

[16] Franz S and Peliti L 1997 J. Phys. A: Math. Gen. 304481

[17] Neher R A and Shraiman B I 2009 Proc. Natl. Acad. Sci. USA 106 6866-6871

[18] Park S C and Krug J 2008 JSTAT: J. Stat. Mech.: Theory and Experiment P04014

[19] Nowak M A 1995 J. Acq. Imm. Def. Syndr. and Human Retrovir. 10 S1-S5

[20] Hallatschek O and Nelson D R 2007 Theor. Pop. Biol. 158-170 